\title{
Anti-LPS Test Strip for the Detection of Food Contaminated with Salmonella and E. coli
}

\author{
Alvin Wang ${ }^{1 *}$, Gabriel Molina ${ }^{1}$, Victor Prima ${ }^{1}$ and Kevin K.W. Wang ${ }^{1,2 *}$ \\ ${ }^{1}$ Center of Innovative Research, Banyan Biomarkers Inc., 12085 Research Drive, Alachua, FL, 32615, USA \\ 2University of Florida, Gainesville, 100 Newell Drive, FL 32610, USA
}

\begin{abstract}
Enterobacteria such as E. coli and Salmonella release an endotoxin, or lipopolysaccharide (LPS) that contributes to food poisoning symptoms. We hypothesize that LPS could be detected by a simple strip test, thus detecting the presence of enterobacteria in food. Here we described the development of a novel Anti-LPS detection strip test. Flexible polystyrene test strips with an absorbent poly (vinylidene fluoride) membrane adhered onto one end were exposed to various dilutions of food or bacterial sources. This was followed by the sequential incubation with a primary anti-LPS antibody, biotinylated secondary antibody, and streptavidin-linked enzyme alkaline phosphatase. The test strip was then developed with substrate 5-bromo-4-chloro-3-indolyl phosphate/nitroblue tetrazolium. Thus, the presence of purple precipitate product would indicate the presence of LPS and enterobacteria. The intensity or darkness of the color was quantified densitometrically and compared to LPS standard curve. The test strip assay was shown to readily detect as low as $25 \mathrm{ng} / \mathrm{mL}$ of purified LPS. In addition, theanti-LPS test strip assay can sensitively detect and quantify LPS released by live E. coli into culture media. Finally, three food groups (strawberry slices, spinach leaves and ground beef) were inoculated with E. coli for two time points at room temperature and then rinse water from each food preparation was subjected to the anti-LPS test strip assay. For all three food groups, the test strip assay can readily detect and quantify both $8 \mathrm{~h}$ and $24 \mathrm{~h}$ bacteria contamination over their respective uncontaminated controls. In conclusion, a simple prototype anti-LPS strip test was developed to readily detect enterobacteria contamination of common food
\end{abstract}

Keywords: Food poisoning; Lipopolysaccharide; Enterobacteria; Strip test; Diagnostics

\section{Introduction}

Escherichia coli (E. coli) belong to a group of pathogenic bacteria called gram-negative enterobacteria that also includes Salmonella and Pseudomonas. E. coli and Salmonella found in contaminated foods can cause diarrhea, and even death in severe cases. E. coli O157:H7 infects over 70,000 Americans and kills approximately 60 annually. Every year, an estimated 1.4 million people are infected by Salmonella and 600 die in the United States. Together, E. coli and Salmonella account for more than $33 \%$ of all food-borne deaths [1]. E. coli causes a battery of diseases in which bacteria cause bloody diarrhea, or it leaves the intestine causing hemolytic uremic syndrome or thrombotic thrombocytopenic purpora (caused by E. coli O157:H7). On the other hand, Salmonella mainly causes gastroenteritis, a condition that is confined to the intestine and can be eliminated more readily. E. coli and Salmonella contamination is especially common with certain household food products. These food items include but are not limited to: undercooked ground beef, vegetables, fruit that was fertilized with cow manure or has been washed with E. coli contaminated water, and fruit juices that have not been pasteurized. When digested, E. coli contaminated food can cause watery diarrhea. Other more severe and rare diseases are kidney failure, blindness, paralysis, Necrotizing Entero Colitis (NEC) and even death [2].

A Lipopolysaccharide (LPS) is a constituent of the outer membrane of the cell wall of certain types of gram-negative bacteria, such as, E. coli, Salmonella, Shigella, Pseudomonas, Neisseria, Haemophilus, and some other lesser known pathogens. While growing, enterobacteria releases small amounts of endotoxins, most of the endotoxins stay on the cell wall until the bacterium disintegrates. Endotoxins are also heat stable, so even boiling the infected food for 30 minutes will not denature it. Watery diarrhea is, in fact caused by released LPS that interacts with the digestive track intestine. Bloody diarrhea, or dysentery, a more severe infection caused by Salmonella, occurs in the colon. Here, cells and tissue are destroyed by the LPS and inflammation does occur. Lastly, LPS is also a causal factor of NEC (necrotizing enterocolitis), a disorder found mostly in newborn infants [2]. If LPS gains entry to the bloodstream, it can bind the host cells, such as macrophages, through the CD14 receptors [3,4], triggering a cascade of adverse systemic responses and organ failure (septic shock) $[5,6]$. In the United States, there are an estimated 751,000 cases of severe sepsis every year [2].

Thus, we hypothesize that if one can develop a simple strip test to detect the presence of enterobacterial contamination of food, it can be used to detect food supply contamination. While bacterial culture tests are readily available, they are laborious and will need a long incubation period before the results are available. We further submit that it is especially important for developing countries where food contamination is widespread.

\section{Materials and Methods}

\section{Materials}

The primary antibody to LPS is an unconjugated mouse monoclonal anti-LPS antibody (Immunoglobin G) and was raised against the carbohydrate core of LPS (Clone WN1 222-5; Cat. \# HM6001; Hycult

*Corresponding authors: Alvin Wang and Kevin Wang, Center of Innovative Research, Banyan Biomarkers Inc., 12085 Research Drive, Alachua, FL, 32615, USA, E-mail: alvin7@gmail.com, kwang@banyanbio.com

Received January 02, 2011; Accepted March 29, 2011; Published March 31, 2011

Citation: Wang A, Molina G, Prima V, Wang KKW (2011) Anti-LPS Test Strip for the Detection of Food Contaminated with Salmonella and E. coli. J Microbial Biochem Technol 3: 026-029. doi:10.4172/1948-5948.1000046

Copyright: (c) 2011 Wang A, et al. This is an open-access article distributed under the terms of the Creative Commons Attribution License, which permits unrestricted use, distribution, and reproduction in any medium, provided the original author and source are credited. 
biotechnology bv, Uden, the Netherlands). This antibody is specific to LPS, and does not cross-react with lipid A or other bacterial protein antigens.Highly purified Lipopolysaccharides (LPS) were from Salmonella enterica serotype typhimurium (Sigma, \#L6511) and from Escherichia coli (E coli) 0127:B8 (Sigma, \#L3129), respectively. The secondary antibody is biotylated sheep anti-mouse immunoglobin G-whole molecule (GE Lifescience; \# RPN1001) and Streptavidin Alkaline Phosphatase (GE Lifescience, \#RPN4202). Substrate is BCIP/ NBT Phosphatase Substrate System (3-Component) (KPL, \# 50-81-00).

Preparation of dilutions of purified LPS samples: A serial dilution of $250 \mu \mathrm{g} / \mathrm{mL}, 100 \mu \mathrm{g} / \mathrm{mL}, 25 \mu \mathrm{g} / \mathrm{mL}, 10 \mu \mathrm{g} / \mathrm{mL}, 2.5 \mu \mathrm{g} / \mathrm{mL}$, $1 \mu \mathrm{g} / \mathrm{mL}, 250 \mathrm{ng} / \mathrm{mL}, 100 \mathrm{ng} / \mathrm{mL}, 25 \mathrm{ng} / \mathrm{mL}, 0$ (none) in Tris-buffer saline with $0.02 \%$ Tween-20 (TBST) was made for each (in 4 replicates). Five hundred micro liter of each dilution sample was added to open-top straight-walled micro centrifuge tube for exposure to anti-LPS test strip (Figure 1, inset).

Preparation of E. coli conditioned media: E. coli (DL21 DE3 strain, Invitrogen Co.)or Bacillus subtilis (B. subtilis) (ATCC 13953) cultureswere grown overnight respectively in two $50 \mathrm{ml}$ tubes containing LB Broth [Luria-Bertani Broth (10 grams of tryptone, 5 grams of yeast, and 10 grams of $\mathrm{NaCl}$ in 1 liter of distilled water)]. The tubes were then centrifuged at 3,000 rpm for 3 minutes and the supernatant (cellconditioned media containing the LPS) was then saved. Dilutions of the cell conditioned media was made with TBST of $1,1 / 3,1 / 10,1 / 30,1 / 100$, $1 / 300$, and 0 (none) from 4 replicate sets of culture. Five hundred micro liter of each dilution sample was added to open-top straight-walled micro centrifuge tube for exposure to anti-LPS test strip. The intensity of purple color was then compared to LPS standard curve (Figure 1).

Food contamination rinse water preparation: Ground beef, spinach leaves, and strawberries were acquired from a local grocery store and rinsed. Each food item was cut and divided into 4 equal portions and each one was inserted into a single tray and labeled for the presence or absence of bacteria $(+\mathrm{E}$ or $-\mathrm{E})$ and the amount of time to be incubated $(8 \mathrm{~h}$ or $24 \mathrm{~h})$. E. coli cell pellet $(200 \mu \mathrm{L})$ was diluted with $13 \mathrm{ml}$ of distilled water and $2 \mathrm{ml}$ of this solution was added to all of the trays labeled " $+\mathrm{E}$ ". While $2 \mathrm{ml}$ distilled water was added to all

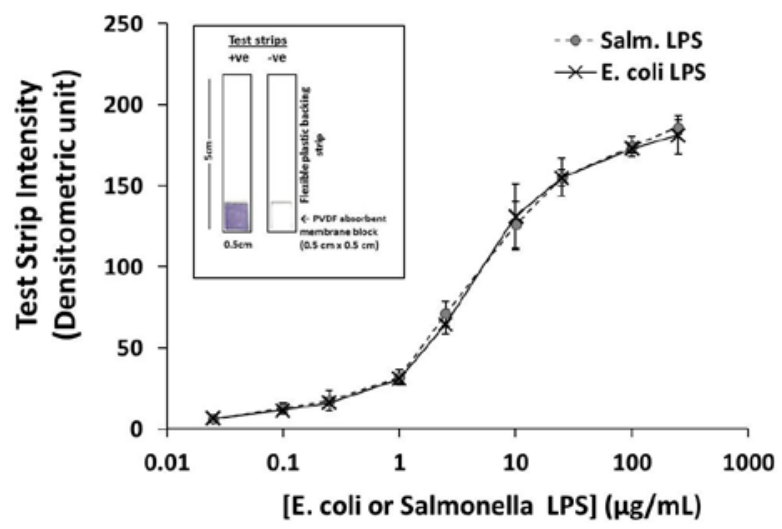

Figure 1: Detection of Purified E. coli and Salmonella LPS titration with Test Strips. The anti-LPS test strips could readily detect both E. coli LPS and Salmonella as low as $0.025 \mu \mathrm{g} / \mathrm{mL}$ with consistency. The assay has a dynamic range that extends from 0.025 to $250 \mu \mathrm{g} / \mathrm{mL}$. Using sigmoidal fit, standard curves can be generated in this fashion for unknown determination. Inset is Image of test strip and dimensions are indicated on the right. Positive (+ve) indicated a developed test strip with strong LPS positive signal, negative (-ve) indicates negative for LPS. the trays labeled "-E". All trays were incubated in the orbital shaker at $24^{\circ} \mathrm{C}$. At $8 \mathrm{~h}$ or $24 \mathrm{~h}$ after incubation, food trays were retrieved from the shaker. To retrieve the rinse water from incubated food, the food items of each tray were collected with forceps and placed into separate $50 \mathrm{ml}$ conical tubes (with cap). Eight $\mathrm{mL}$ distilled water was added into each tube with gentle shaking. The rinse solution was then collected with a transfer pipette. Original rinse solutions $(500 \mu \mathrm{L}$ for each condition $(4$ replicate sets) were then diluted $1 / 3$ with distilled water before being transferred into the micro centrifuge tubes $(500 \mu \mathrm{L})$. Tween-20 $(0.25$ $\mu \mathrm{L}$ ) was also added into each micro centrifuge tube to achieve a final concentration of $0.05 \%$.

Anti-LPS test strip assay: The anti-LPS test strip was designed with the following principles: (i) simplicity of operation, (ii) high sensitivity, (iii) low background signal; and (iv) providing qualitative and quantitative data. After some optimization, an anti-LPS detection strip test was developed. Prototype flexible semi-rigid test strips ( 0.5 $\mathrm{cm}$ wide $\mathrm{x} 5 \mathrm{~cm}$ long) with an absorbent membrane (PVDF) $(0.5 \mathrm{~cm}$ $\mathrm{x} 0.5 \mathrm{~cm}$ ) adhered onto one end (Figure 1, inset).PDVF-part of test strips were pre-wet for 5 seconds in $100 \%$ methanol, followed by TBST. Singlestripswere placed into individual straight-walled microfuge tubes with test samples and allowed to incubate on the shaker for $1 \mathrm{~h}$ at room temp. Strips are taken out and quickly rinsed with TBST with transfer pipette $(2 \mathrm{~mL}$ each) and further soaked in $10 \mathrm{~mL}$ TBST in buffer trays for $2 \mathrm{~min}$, followed by TBST with $10 \mathrm{~mL}$ of blocking solution (TBST with $5 \%$ skim Milk) for $30 \mathrm{~min}$. This is replaced with primary anti-LPS solution (1/1,000; Mouse Anti-LPS carbohydrate LPS core monoclonal antibody) in TBST-milk and strips are further incubated on shaker for $1 \mathrm{~h}$ and then rinsed and soaked with TBST as above. TBST was then replaced with $10 \mathrm{ml}$ of biotinylated secondary antibody $(1 / 1,000$ in TBST-5\% Milk) and incubated for $1 \mathrm{~h}$. TBST rinsing was repeated and followed with a streptavidin alkaline phosphatase solution $(1 / 2,000)$ (TBST-5\% Milk) incubation (30 min. After final rinsing, test strips were developed in a shallow tray with $10 \mathrm{~mL}$ substrate solution (BCIPNBT (KPL; Cat. \#: 50-81-00) for exactly $15 \mathrm{~min}$. The product is a purple precipitate that deposited onto the test strip. Thus, the presence of purple color of the PVDF membrane-block would indicate the presence of LPS (Figure 1, inset). Reaction is stopped by putting the strip in Distilled water and then air-dried. When quantification data is needed, fully dried strips were scanned with a scanner and strip density analyzed with Image J software. LPS concentration in sample can be further calculated with the use of a LPS (E. coli) standard curve.

\section{Results}

\section{Anti-LPS test strip design and work flow}

Using the anti-LPS test strip we developed, the presences of purple color of the PVDF membrane-block would indicate the presence of LPS (Figure 1, inset). When the color intensity was then plotted against the concentrations of LPS, we found that the test strip can readily detect as low as $0.025 \mu \mathrm{g} / \mathrm{mL}$ (or $25 \mathrm{ng} / \mathrm{mL}$ ) of LPS from both E. coli and Salmonella while maintaining a dynamic range up to $200 \mu \mathrm{g} / \mathrm{mL}$ (Figure 1). Using sigmoidal fit, standard curves can be generated in this fashion for unknown determination (Figure 1).

\section{Anti-LPS test strip detection of LPS released from $E$. coli into culture media}

In this experiment, various dilutions of $\mathrm{E}$. coli conditioned media were subjected to anti-LPS test strip assays and compared to the standard curve established in Figure 1. The results show that test strip assays can readily detect and quantify LPS released by live E. coli culture, even with $1 / 300$ dilution of media ( $p<0.05$, Student T-test) (Figure 
2). In contrast, cell conditioned media from gram-positive bacteria $B$. subtilis (which does not produce LPS) gave no detectable LPS signals at all dilutions when tested on this test strip assay, demonstrating the specificity of this assay (Figure 2). Taken together, results in Figure 1 and 2 shows that the anti-LPS strip test is very sensitive in detecting enterobacteria released LPS quantitatively.

\section{Testing of rinse water from $E$. coli contaminated food}

In this set of experiments, we inoculated three different food groups (ground beef, spinach leaves and strawberry slices) with E. coli for two time points ( $8 \mathrm{~h}$ and $24 \mathrm{~h}$ ) at room temperature and then fixed volume of rinse water from each type of food ( $n=4$ for each test group) was prepared (see Methods). Rinse water (1/3 diluted) from each group wasthensubjected to anti-LPS test strip assay and quantified against LPS standard curve.

For the ground meat group, the test strips detected no signal from rinse water of ground beef not contaminated with bacteria (Figure $3 \mathrm{~A})$. On the other hand, the test strip assay detected LPS release $(27.6 \mu \mathrm{g} / \mathrm{mL})$ from $8 \mathrm{~h}$ bacteria contamination over their respective uncontaminated controls ( $\mathrm{p}=0.049$, Student Test) (Figure 3A). By 24 $\mathrm{h}$ of E. coli contamination, the LPS release rose drastically to $468.2 \mu \mathrm{g} /$ $\mathrm{mL}$ over controls $(\mathrm{p}=0.0002)$.

Similarly, for the spinach leaf group, the test strips detected no signal from rinse water of spinach leaves not contaminated with bacteria (Figure 3B). Yet, the test strip assay detected LPS release $(397.8 \mu \mathrm{g} / \mathrm{mL}$ ) from $8 \mathrm{~h}$ bacteria contamination over their respective uncontaminated controls ( $\mathrm{p}<0.0001$, Student Test). By $24 \mathrm{~h}$ of E. coli contamination, the LPS release rose to $835.7 \mu \mathrm{g} / \mathrm{mL}$ over controls $(\mathrm{p}<0.0001)$ (Figure $3 \mathrm{~B})$.

Lastly, for the strawberry group, again, the test strips detected no signals from rinse water of strawberry slices not contaminated with bacteria (Figure $3 \mathrm{C}$ ). In contrast, the anti-LPS test strip assay detected LPS release $(106.0 \mu \mathrm{g} / \mathrm{mL})$ from $8 \mathrm{~h}$ bacteria contamination over their respective uncontaminated controls ( $\mathrm{p}<0.0001$, Student Test), while the LPS release rose to $250.3 \mu \mathrm{g} / \mathrm{mL}$ by $24 \mathrm{~h}$ of contamination over controls $(\mathrm{p}=0.004)$ (Figure 3C). Thus, we have developed a simple antiLPS strip test and demonstrated that it can readily detect enterobacteria contamination of common food.

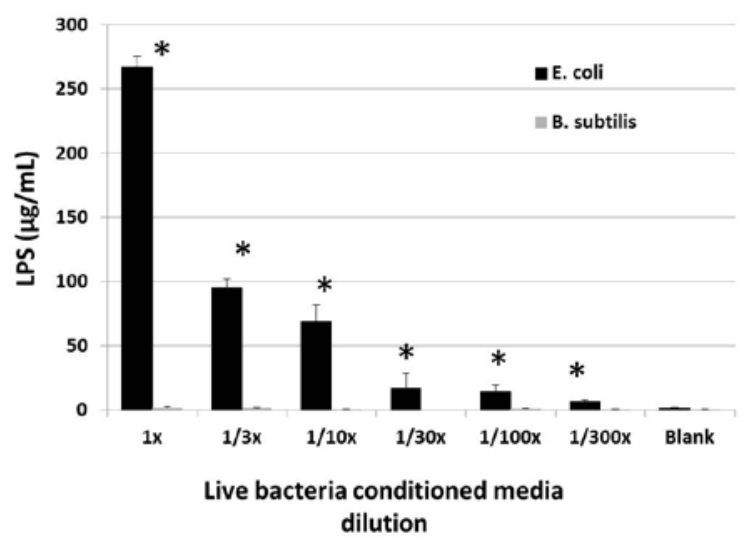

Figure 2: Detection of Live E. coli conditioned culture media with antiLPS test strips. With a serial dilution of E. coli or B.subtilis conditioned culture media (LB broth) was subjected to anti-LPS strip test. All dilutions (down to $1 / 300 x)$ have detectable levels of LPS. ( ${ }^{*} p<0.05$, Student T-test) when compared to control (blank).
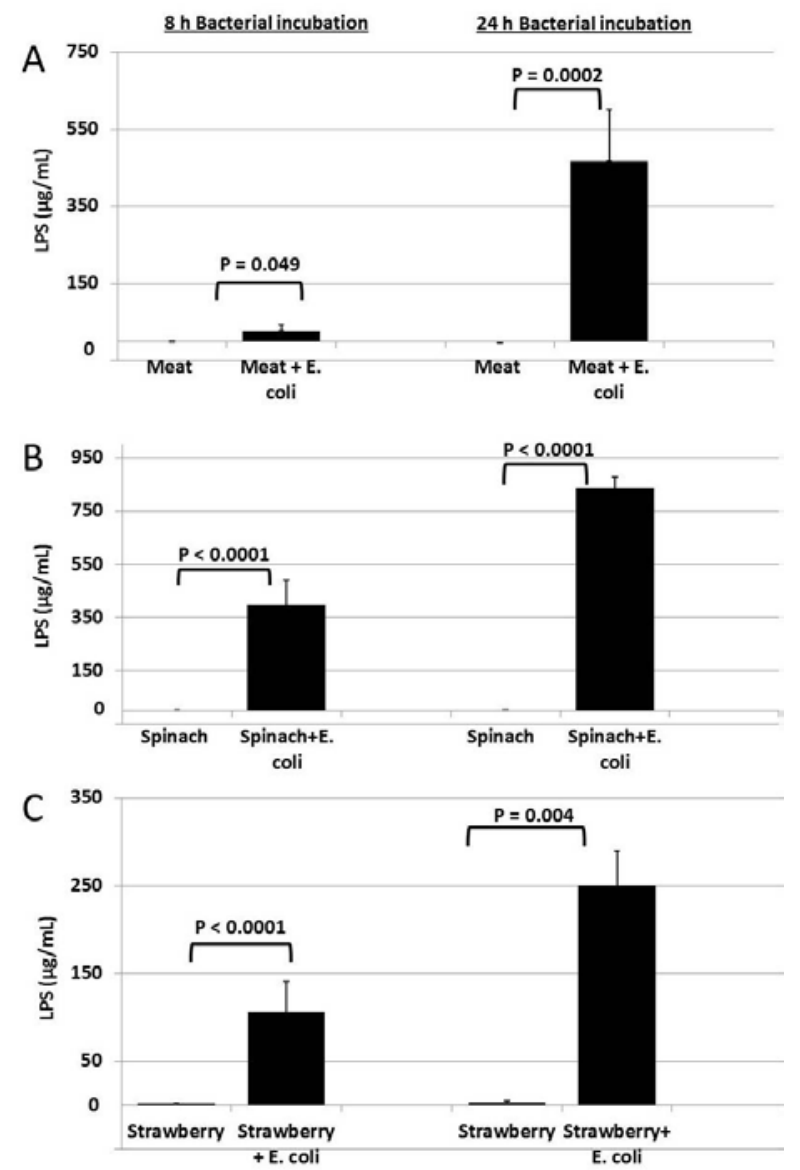

Figure 3: Detection of LPS in rinse solution from E. coli contaminated grounded meat, spinach leaves and strawberry slices. (A) For ground beef, the strips could detect LPS signals from the rinse water ( $1 / 3$ diluted) after $8 \mathrm{~h}(\mathrm{p}=0.049$, Student's Test-test) and $24 \mathrm{~h}$ of bacteria incubation $(p<0.002)$, when compared to their respective no bacteria control. (B) For spinach leaves, the test strips can detect LPS signals from the rinse water ( $1 / 3$ diluted) after $8 \mathrm{~h}$ $(p<0.0001$, Student's Test-test) and $24 \mathrm{~h}$ of bacteria incubation $(p<0.0001)$, when compared to their respective no bacteria control. (C) For strawberry slices, the test strips can detect LPS signals from the rinse water $(1 / 3$ diluted after $8 \mathrm{~h}(\mathrm{p}<0.0001$, Student's Test-test) and $24 \mathrm{~h}$ of bacteria incubation ( $\mathrm{p}=$ $0.04)$, when compared to their respective no bacteria control.

\section{Discussion}

Lipopolysaccharide from the outer membrane of gram-negative enterobacteria is strongly associated with septic shock. Sepsis can be defined as a clinical condition, or an immune response to infection, characterized by systemic inflammation and coagulation [6]. Binding of LPS via LPS-binding protein (LBP) to the CD14 receptor on the cell membranes host cells (e.g. macrophages) results in hyper-activation of inflammatory cells and excessive production of pro-inflammatory cytokines, including TNF- $\alpha$, IL- 6 and C-reactive protein $[3,7$, and 8]. Tissue injury and multiple organ failure often follow sepsis and contribute to high morbidity and mortality rates in intensive care units $[6,9,10,11]$.

Enterobacteria (most commonly E coli and Salmonella) food poisoning occurs annually and can post significant threats to human health, as well as create a burden to the health care system. The purpose of this study was to develop a simple and portable test that detects whether food is contaminated with bacteria (i.e. E. coli and Salmonella). 
Since enterobacteria release LPS, which should be detected by an antibody-based method, we hypothesized that this method can be used to develop a simple test for bacterial contamination of food. Our results showed that the test strips created were (i) able to detect and quantify LPS originated from both E. coli and from Salmonella bacteria as low as $0.1 \mu \mathrm{g} / \mathrm{mL}$ (Figure 1), (ii) sensitive enough to detect LPS released by live E. coli diluted in 1/300 (Figure 2), and specific to Gram negative bacteria (e.g. E. coli) over Gram positive bacteria (e.g. B. subtilis) and (iii) able to detect the presence of E. coli-contamination in three types of food at two time points (Figure 3). Overall, the foods incubated with the bacteria for $24 \mathrm{~h}$ yield higher LPS signals than those incubated with the bacteria for $8 \mathrm{~h}$. Importantly, the test strips detected little or no signal from all three food extracts that were not contaminated with bacteria. The level of LPS that is toxic to humans was reported to be around 275 $\mu \mathrm{g} / \mathrm{mL}[1]$, which is well within the detection range of our test strips. We also found that extract from bacteria contaminated ground beef $(24$ h) and spinach bacteria (both $8 \mathrm{~h}$ and $24 \mathrm{~h}$ ) have reached LPS levels at $300-500 \mu \mathrm{g} / \mathrm{mL}$, which is considered toxic to humans and unsafe for consumption.

It is noted that other bacterial detection methods do exist; including culturing the bacteria, but it can take up to 24-48 h for a definitive result [17]. Alternatively, ELISA for a specific bacteria antigen has been reported in the literature $[12,13,14]$. However, the drawback for this approach is that it will only specifically detect one type of bacteria and ELISA assay requires dedicated equipment such as ELISA plate-reader, plate-washer and skilled technical support. Keen and Mitchell [15] also described a strip test that monitors nitrate reduction by bacteria. However, it is subjected to interference by enzymes and other substances. We submit that the anti-LPS strip test assay described here will be a complementary enterobacteria screening method for food safety. Since LPS is released form bacteria and is a causative agent for food poisoning, it provides both signal amplification and a quantitative assessment of risk. It is also important to point out that we have confirmed that our strip test assay can equally detect both LPS purified from E coli and Salmonella, but not Gram positive bacteria. We acknowledge that there are many other LPS-bearing Enterobacteria that we have not tested. However, LPS structure is virtually identical among all Enterobacteria [16]. Thus, it is reasonably assumed that it will be detected with our current strip test. We believe these additional experiments, although beyond the scope of our current communications, should be pursued.

In conclusion, we developed a novel anti-LPS test strip assay to detect the presence of bacterial contamination in food that can potentially be used to screen for food contamination. It is noted that what we have demonstrated is only a proof-of-principle prototype assay. Further optimizations will clearly be needed. These include (i) simplifying the assay step and reducing assay time by direct coupling of detecting enzymes to the anti-LPS antibody, (ii) standardizing the strip and PDVF membrane manufacturing for further reduce variability, and (iii) further increasing the detection sensitivity by selecting antiLPS antibody with even higher affinity. We also envision that, based on existing technology, a companion point-of-care or hand held reader can be used to quantify the level of LPS detected on the test strip with a built-in internal standard.

\section{Acknowledgement}

The authors would like to acknowledge Dr. Stan Svetlov for his helpful scientific discussion and encouragement.

\section{References}

1. CDC, Division of Foodborne, Bacterial and Mycotic Diseases, Centers for Disease Control and Prevention (2006) E. coli, http://www.cdc.gov/nczved/ dfbmd/disease_listing/stec_gi.html Retrieved Dec, 9, 2010

2. Chan KL, Wong KF, Luk JM (2009) Role of LPS/CD14/TLR4-mediated inflammation in necrotizing enterocolitis: pathogenesis and therapeutic implications. World J Gastroenterol. 15: 4745-4752.

3. Dobrovolskaia MA, Vogel SN (2002) Toll receptors, CD14, and macrophage activation and deactivation by LPS. Microbes Infect 4: 903-914.

4. Moore KJ, Andersson LP, Ingalls RR, Monks BG, Li R, et al. (2000) Divergent response to LPS and bacteria in CD14-deficient murine macrophages. J Immunol 165: 4272-4280.

5. Hewett JA, Roth RA (1993) Hepatic and extrahepatic pathobiology of bacteria lipopolysaccharides. Pharmacol Rev 45: 382-411.

6. EP McIntyre L, Morro DC, Rivers KK (2005) early and innovative interventions for severe sepsis and septic shock: taking advantage of a window of opportunity. CMAJ 173: 1054-1065

7. Castelli GP, Pognani C, Meisner M, Stuani A, Bellomi D, et al. (2004) Procalcitonin and C-reactive protein during systemic inflammatory response syndrome, sepsis and organ dysfunction. Crit Care 8: R234-242.

8. Rosenfeld Y, Shai Y (2006) Lipopolysaccharide (Endotoxin)-host defense antibacterial peptides interactions: role in bacterial resistance and prevention of sepsis. Biochim Biophys Acta 1758: 1513-1522.

9. Beutler B, Cerami A (1988) Tumor necrosis, cachexia, shock, and inflammation: a common mediator. Annu Rev Biochem 57: 505-518.

10. Knotek M, Rogachev B, Wang W, Ecder T, Melnikov V, et al. (2001) Endotoxemic renal failure in mice: Role of tumor necrosis factor independent of inducible nitric oxide synthase. Kidney Int 59: 2243-2249.

11. Lee RP, Wang D, Lin NT, Chen HI (2002) Physiological and chemical indicators for early and late stages of sepsis in conscious rats. J Biomed Sci 9: 613-621.

12. Zamora BM, Hartung M (2002) Chemiluminescent immunoassay in comparison with the indirect ELISA as reference method for detecting Salmonella antibodies in swine meat juice. Berl Munch Tierarztl Wochenschr. 115: 369-380.

13. Wang L, Liu B, Kong Q, Steinrück H, Krause G, et al. (2005) Molecular markers for detection of pathogenic Escherichia coli strains belonging to serogroups $O$ 138 and $O$ 139. Vet Microbiol 111: 181-190.

14. Kich JD, Schwarz P, Eduardo Silva L, Coldebella A, Piffer IA, et al. (2007) Development and application of an enzyme-linked immunosorbent assay to detect antibodies against prevalent Salmonella serovars in swine in southern Brazil. Journal of Vet Diagn Invest 19: 510-517.

15. KeenAP, Mitchell RG (1986) Commercial strip test for reduction of nitrate by bacteria. J Clin Pathol 39: 118.

16. Rietschel ET, Kirikae T, Schade FU, Ulmer AJ, Holst O, et al. (1993) The chemical structure of bacterial endotoxin in relation to bioactivity. Immunobiology 187 169-190.

17. Fox A (2008) Bacteriology - Chapter two cultures and identification of infectious agents; Microbiology and Immunology On-line. http://pathmicro.med.sc.edu/ fox/culture.htm. Retrieved Dec 15, 2010. 